\title{
Joint user association and interference mitigation for drone-assisted heterogeneous wireless networking
}

\author{
Chuan'an Wang ${ }^{1,2}$, Bo Hu$^{1 *}$ and Shanzhi Chen ${ }^{3}$
}

\begin{abstract}
Using drone as aerial access point (AAP) to provide communication connectivity becomes an attractive application in future industrial wireless networking. However, the user association becomes more complex in the 3-tier droneassisted heterogeneous networking (DAHN) due to the various types of APs. To address the issue, we formulated it as an association degree maximization problem, in which the load of APs and the user quality of service (QoS) rate requirement are considered. Since this is a non-linear mixed integer optimization problem, we relax the associated index variable and adopt the distributed heuristic method to find the near-optimal solution. Further, to mitigate the intra- and inter-layer interference, the coordinated multiple points (CoMP) supporting approach for edge user is used for enhancing the service performance. Simulations show that the proposed scheme provides better performances with a significant complexity reduction.
\end{abstract}

Keywords: User association, Interference mitigation, Drone, Heterogeneous wireless network

\section{Introduction}

At present, it is going to be a new tendency that the advanced wireless communication techniques are introduced to the industrial field. As more user equipment (UE) are connected to the network, dense deployment of lowpower access points (LAPs) is one intuitive way to handle the ever-increasing data traffic demands [1]. However, current APs are deployed in certain geographical locations according to long-term traffic behaviors and lack flexibility in redeployment. Moreover, such rigid access network is hardly to handle the difficult-to-predict traffic patterns caused by the temporal and spatial variations in user densities and user application rates. At the same time, with the development of drone communication technology, the drone used as aerial AP (AAP) becomes an attractive application in future wireless communications. Therefore, to enhance access network flexibility for supporting massive dynamic connections, the 3-tier drone-assisted heterogeneous networking (DAHN) is a promising solution [2]. While there are several advantages for the use of AAP,

\footnotetext{
* Correspondence: hubo@bupt.edu.cn

${ }^{1}$ State Key Lab of Networking and Switching Technology, Beijing University of Posts and Telecommunications, Beijing 100081, China Full list of author information is available at the end of the article
}

there are some issues have not been addressed yet in the literature.

One of the fundamental issues is how to associate UEs with an appropriate serving AP. In the traditional network, UE always connect to the strongest AP, which offers either the maximum signal-to-interference-plus-noise ratio (SINR) or the best reference signal received power (RSRP) [2]. However, the association between the UE and the network becomes more complex in DAHN for the following reasons. First, due to a large difference of transmit power between macro AP (MAP), LAP, and AAP, the user association methods mentioned above may lead to unbalance of user distribution between the various types of APs. Moreover, in order to effectively suppress the interand intra-layer interference problem in DAHN, the coordinated multiple points (CoMP) technology is adopted for the edge UEs, which makes the connection between UEs and serving APs more complicated.

In this letter, we will investigate user association and interference mitigation issues for DAHN. For effectively tackling user association problem, we jointly consider the load of each AP and UE's quality of service (QoS) rate requirement instead of only utilizing the SINR or RSRP when designing an association algorithm, and formulate it 
as a UE-AP association degree maximization problem. Further, an edge user CoMP (EU-CoMP) supporting technology is proposed for enhancing the services performance, and a coordinated APs grouping solution is given.

The rest of the paper is organized as follows. In Section 2, we discuss the proposed work in the context of related works. Section 3 presents the system model and basic conceptions. In Section 4, a user association algorithm based on association degree is proposed, and the EU-CoMP supporting approach is adopted. Section 5 presents the performances of the proposed algorithm. Finally, we give a conclusion of this work in Section 6.

\section{Related works}

Numerous excellent contributions have surveyed the user association problem in heterogeneous network (HetNet). The work in [3] investigates joint user association and spectrum allocation optimization issues for a 2-tier HetNet, and optimization problem is formulated as a mixedinteger non-linear programming problem. In [4], the authors aim to optimize the user association with various user priorities to solve the load balancing problem. Rui Han et al. solve the user association problem by introducing the topological potential between UEs and APs in [2]. Based on the consideration of the load of each AP and user's achievable rate, the work in [5] proposes a loadaware and QoS-aware user association strategy. The authors in [6] focus on maximization of user satisfaction with provided data rates and propose an algorithm that associates users with the most suitable LAP and AAP.

In the user association problem conjunction with interference coordination, the authors in [7] formulate the combinatorial optimization problem as a networkwide utility maximization problem. Further, they reveal that the optimal blank sub-frame density is the ratio of the number of vulnerable UEs and total UEs. In [8], the problem is formulated as a proportional fair-based joint optimization problem, in which the bandwidth ratio of protected radio resources is used for inter-cell interference coordination. An energy-efficient management framework is built on characterizing the interference coupling by predefined interference patterns in [9], and a tailored algorithm is developed to solve the formulated problem. In [10], a centralized heuristic algorithm is proposed to discover the optimal almost blank subframe, which is used to maximize the number of served UEs while ensuring interference management.

Although the above proposals have addressed the problem of user association in HetNets, no other works have provided a comprehensive view of this problem in the 3tier DAHN. The objective of this paper is to give an efficient association strategy for balancing the number of severing UEs between the various types of APs while satisfying the UE's QoS rate requirements.

\section{System model}

We consider a downlink DAHN where the control/signaling plane and user plane (CP/UP) are decoupled at the air interface, as shown in Fig. 1. As the entity center of the CP, MAP can possess the global system information and handles UE connectivity as well as different radio-specific functions, and it could also provide UP services for some UEs with low-speed data requirements. The LAP is mainly used as a UP entity to provide UEs with data transmission and resource dynamic allocation, while the AAPs can offer improved capacity in hotspot areas and offload some traffic from the ground APs. For simplicity, denoted by $\mathscr{B}=\{\{0$ \}$\cup \mathcal{D} \cup \mathscr{L}\}$ as the set of all APs, where 0 represents MAP, $\mathcal{D}$ and $\mathscr{L}$ represent the set of AAPs and LAPs, respectively. UEs are randomly distributed in the coverage area of the MAP, and the set of UEs is denoted by $\mathcal{U}=\{1,2, \cdots, U\}$.

We define $g_{u, b}$ as the channel gain from the transmitting AP $b$ to the receiving UE $u$, where we consider twochannel models. When $b \in \mathcal{D}$, the transmission channel can be expressed by a generic air-to-ground (AtG) path loss model studied in [11], and $g_{u, b}$ is obtained by AtG path loss model. Another channel model can be modeled as a Rayleigh fading channel when $b=\{0\}$ or $b \in \mathscr{L}$, and $g_{u, b}$ contains distance-dependent path loss and small-scale Rayleigh fading [12].

\section{User association and interference mitigation}

In DAHN, the UE first establishes radio resource control (RRC) connection with the MAP through the CP interface, and then sends a business request for establishing UP connection. A UE will choose the most attractive AP as its serving AP with the assistance of MAP. According to [13], the lower power of AP, the smaller access chance for UEs. So an intelligent user association policy in DAHN should depend on both the SINR and the load.

\subsection{User association strategy based on association degree}

Obviously, UEs prefer to associate with the APs that can meet the guaranteed QoS rate requirement and have lower load. Thus, the objective of the proposed association strategy is to balance the number of severing UEs between the various types of APs while satisfying the UE's QoS rate requirements.

Given the power $\sigma^{2}$ of the additive white Gaussian noise, the SINR of transmission link between AP $b$ and $\mathrm{UE} u$ on one resource block (RB) is given by

$$
\gamma_{u, b}=\frac{p_{b} g_{u, b}}{\sum_{b^{\prime} \in \mathscr{B} \backslash\{b\}} p_{b^{\prime}} g_{u, b^{\prime}}+\sigma^{2}}
$$

where $P_{b}$ is the given fixed transmit power of the AP $b, \Sigma_{b^{\prime} \in \mathscr{B} \backslash\{b\}} p_{b^{\prime}} g_{u}, b^{\prime}$ is the interference from all the cochannel Aps for UE $u$. 
The corresponding achievable data rate of this transmission is

$$
r_{u, b}=\operatorname{slog}\left(1+\gamma_{u, b}\right)
$$

where $s$ is the bandwidth of one $\mathrm{RB}$, and we assume all the RBs have the same size.

In order to balance the load between all kinds of APs while maintaining the UEs' performance requirements, the association degree is defined in terms of QoS rate requirement and load levels. Then association degree between AP $b$ and UE $u$ can be expressed as

$$
\begin{aligned}
\vartheta_{u, b} & =\exp \left(\frac{r_{u, b}}{\sum_{u \in \mathcal{U}^{x_{u, b}}}}\right) \\
& =\exp \left(\frac{\operatorname{slog}\left(1+\gamma_{u, b}\right)}{\sum_{u \in \mathcal{U}^{x_{u, b}}}}\right)
\end{aligned}
$$

where $x_{u, b}$ is a Boolean variable indicating whether UE $u$ is connected to AP $b, \sum_{u \in \mathcal{U}} x_{u, b}$ is the number of UEs associated with AP $b$, which is also often considered as the load of AP. It is worth noting that each AP is assumed to have at least one UE associated with it, i.e., $\sum_{u \in \mathcal{U}^{X}} x_{u, b} \neq 0$.

According to Eq. (3), we can see that SINR is not the only factor which affects the association procedure. Under the same load, the AP with higher SINR will be more attractive to UEs. AS the candidate AP load becomes too large, the reduced association degree will make it less attractive to UEs, from which a balancing user association can be guaranteed.

The user association problem can be formulated as an association degree maximization problem in consideration of user QoS rate requirements, i.e.,

$$
\begin{aligned}
& \max _{X} \sum_{u \in \mathcal{U}} \sum_{b \in \mathscr{B}} x_{u, b} \exp \left(\frac{r_{u, b}}{\sum_{u \in \mathcal{U}} x_{u, b}}\right) \\
& \text { s.t. } C 1: \quad \sum_{b \in \mathscr{B}} x_{u, b}=1, \quad \forall u \in \mathcal{U} \\
& C 2: \quad x_{u, b} \in\{0,1\}, \quad \forall u \in \mathcal{U}, \forall b \in \mathscr{B} \\
& \text { C3: } \quad r_{u, b} \geq r_{m}, \quad \forall u \in \mathcal{U}, \forall b \in \mathscr{B}
\end{aligned}
$$

where $X=\left\{x_{u, b} \mid u \in U, b \in B\right\}$ is the association matrix, and the constraints in Eq. (4): C1) indicates that any UE can only associate with one AP; C2) denotes that the value range of the variable $x$ is binary; C3) is the UE's QoS rate constraint, where $r_{m}$ is the UE's minimum Qos rate requirement.

Remark that optimization problem (4) is non-convex due to the coupling of Boolean variable and continuous variable, and it is very complex to find the optimal solution by solving the mathematical model. For non-convex problems, a common method is to relax the discrete variables to the continuous value region for the solution.
Thus, we relax the constraint $\mathrm{C} 2$ to $0 \leq x_{u, b} \leq 1$. The problem (4) can be rewritten as

$$
\begin{aligned}
& \max _{X} \sum_{u \in \mathcal{U}} \sum_{b \in \mathscr{B}} x_{u, b} \exp \left(\frac{r_{u, b}}{\sum_{u \in \mathcal{U}^{X}} x_{u, b}}\right) \\
& \text { s.t. } C 1: \sum_{b \in \mathscr{B}} x_{u, b}=1, \quad \forall u \in \mathcal{U} \\
& \text { C2: } 0 \leq x_{u, b} \leq 1, \quad \forall u \in \mathcal{U}, \forall b \in \mathscr{B} \\
& \text { C3: } r_{u, b} \geq r_{m}, \quad \forall u \in \mathcal{U}, \forall b \in \mathscr{B}
\end{aligned}
$$

\subsection{Distributed heuristic solution}

In the relaxed optimization problem (5), the objective optimization function is quasi-concave, while the constraint condition is convex, so the optimal solution exists. However, using the optimal association matching algorithm approach based on the exhaustive to solve the problem (5) tends toward high computational complexity and low reliability, and the complexity is $O\left(U^{|\mathscr{B}|}\right)$, where $|\mathscr{B}|$ is the cardinality of the set $\mathscr{B}$.

To reduce the computational complexity, we give a distributed heuristic algorithm with a near-optimal solution that provides a theoretical performance guarantee. When the MAP receives the UE's UP connection request, the APs that meet the user QoS rate requirement are selected as the candidate service APs set. Then, Then the UE's association degree with each AP in the candidate set is calculated, and the AP with the highest association degree is selected as the final service AP.

Inspired by the physical idea mentioned above, the process of the proposed distributed heuristic user association algorithm is detailed in Algorithm 1.

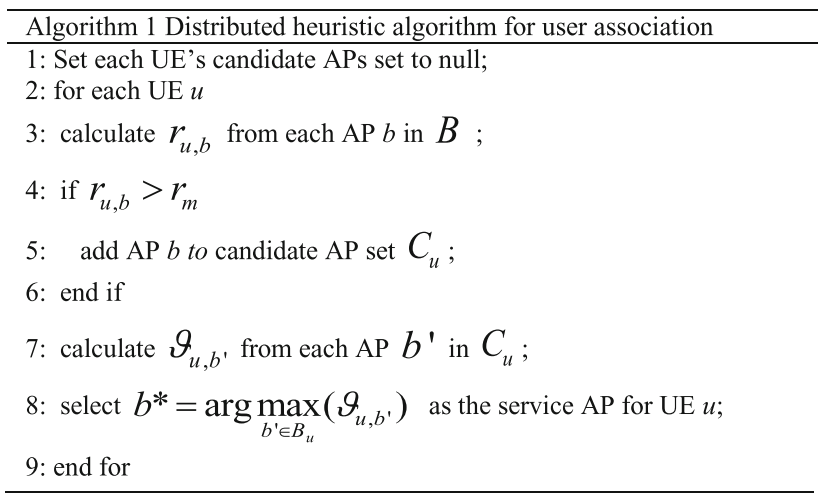

The proposed user association algorithm is a lowcomplexity distributed algorithm with a near-optimal solution, which decomposes the optimal problem with $U^{|\mathscr{B}|}$ possible association combinations into $U$ subproblems. Considering each sub-problem solution, the main computational operations are to calculate the association degree from all the APs for each UE, and the worst complexity is $O(2 *|B|)$. We assume that each UE remains independent, and then the overall complexity of the proposed algorithm is $O(2 * U *|B|)$. 


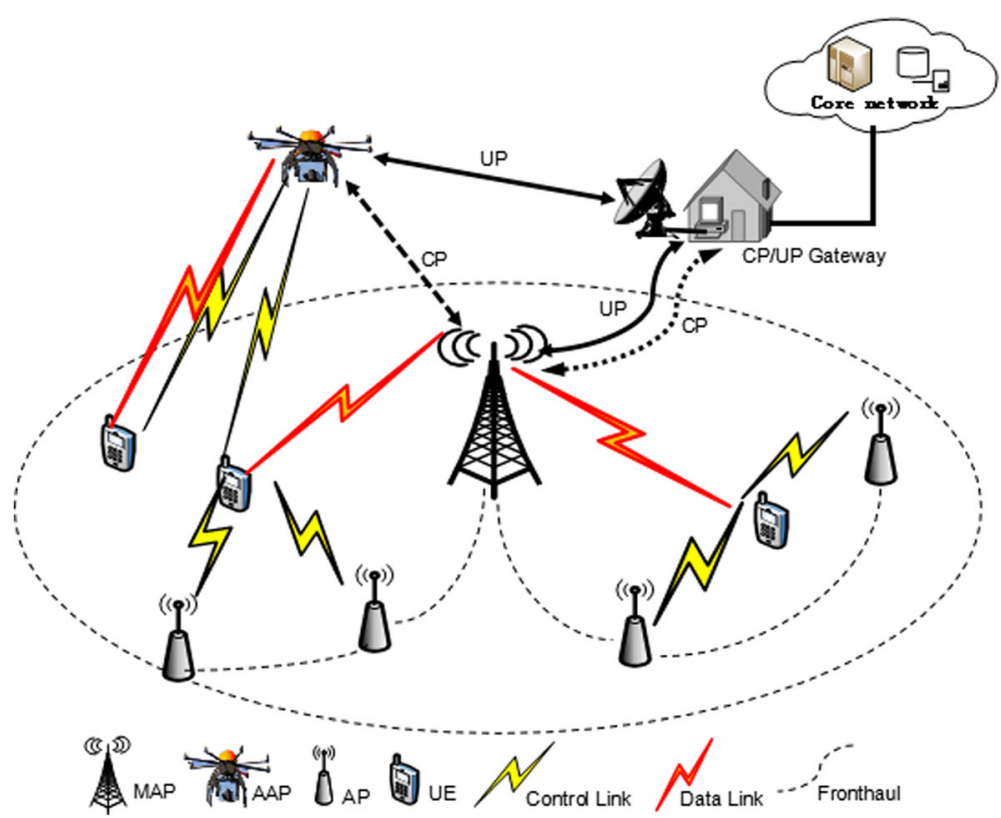

Fig. 1 A 3-tier drone-assisted heterogeneous network

In this letter, the widely accepted Jain fairness index (JFI) $[2,14,15]$ is used to evaluate the fairness of the user association. Due to the different service capabilities of APs in each layer, we limit the JFI calculation to one layer. The JFI for $i$ th $(i \in\{0,1,2\})$ layer can be expressed as:

$$
F I_{i}=\frac{\left(\sum_{j=1}^{B_{i}} N_{U E}^{j}\right)^{2}}{B_{i} \sum_{j=1}^{B_{i}}\left(N_{U E}^{j}\right)^{2}}
$$

where $B_{i}$ is is the number of users in $i$ th layer, and $N_{U E j}$ is the number of UEs associated with $j$ th AP.

\subsection{EU-CoMP supporting technology for interference mitigation}

The service AP can be determined for the UE by using the proposed distributed heuristic user association

Table 1 System parameters used in simulation

\begin{tabular}{ll}
\hline Parameter & Value \\
\hline Frequency & $2.0 \mathrm{GHz}$ \\
System bandwidth & $10 \mathrm{MHz}$ \\
Transmit power & MAP:46 dbm; \\
& AP: $36 \mathrm{dBm} ;$ \\
& AAP:30 dBm \\
Path loss model & AP: $140.7+37.6 \log _{10}(d)$ \\
& MAP: $128.1+36.7 \log _{10}(d)$ \\
Number of UEs & AAP:AtG path loss model [11] \\
QoS rate requirement & $300 \mathrm{UEs}$ randomly distributed \\
\hline
\end{tabular}

algorithm. When the UE is at the edge of the serving AP, the strong intra- and inter-interference will lead to poor service quality. Thus, the EU-CoMP supporting approach is required to improve the service performance of edge UE, which means the APs with strong interference need to be changed to the cooperative services APs. The key component of EU-CoMP approach is to determine which APs collaborate to serve which UEs, which is called a coordinated APs group (CAPG) formation problem. This is also an optimal matching problem [16]. Obviously, a global coordination of all interfering APs is practically impossible. Hence, each edge UE could be cooperatively served by a limited number of APs, and it is also considered as a group-making problem [17].

First, what needs to be done is to determine the edge UEs. When the RSRP received from the service AP does not differ much to the RSRP from the interfering AP, the UE is considered to be in the edge region. This means that if the received RSRP meets the threshold difference, the UE $u$ can be considered as an edge UE and adds it to the edge UEs set $\mathscr{E}$, as shown in the following conditional expression.

$$
\xi_{o, u}-\xi_{b, u} \leq \delta \Rightarrow u \in \mathscr{E}
$$

where $\xi_{b, u}$ is the RSRP received by UE $u$ from the AP $b$ with strong interference, $\xi_{o, u}$ is the RSRP received from the service AP $o$.

It is clear that when the interfering AP is added into CAPG to provide cooperative services to the edge UE, the SINR will increase without the involvement of other 


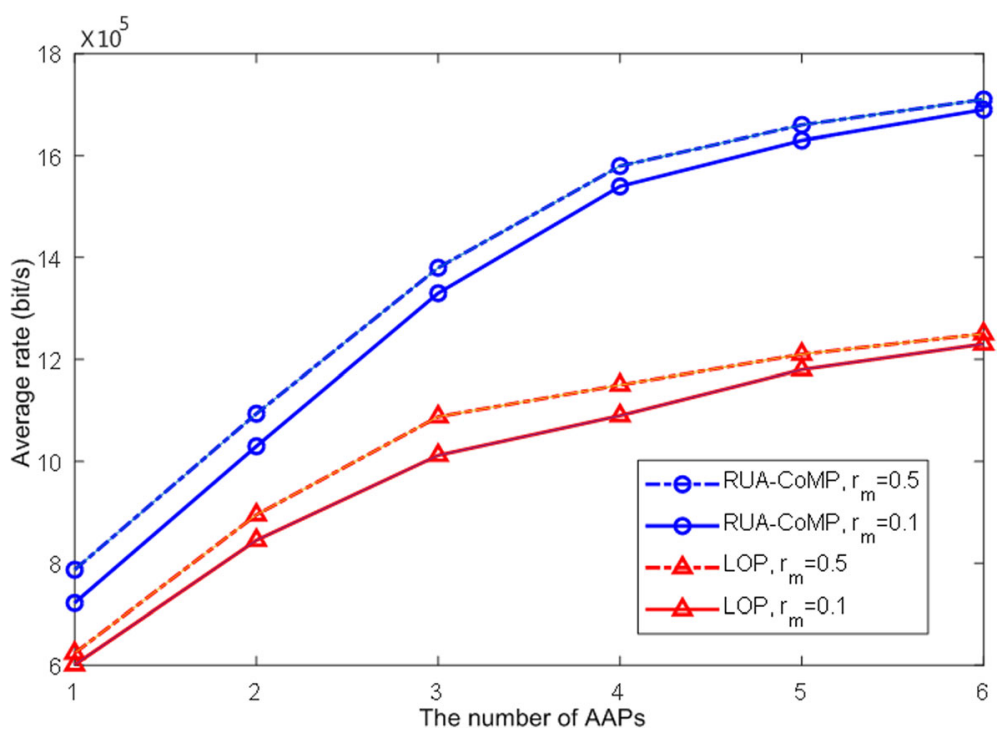

Fig. 2 UE's average data rate under different AAP numbers

UEs, which is called as the cooperation benefit (CB). The CB $v_{u, n}$ of AP $b$ for UE $u$ is given by

$$
v_{u, b}=\gamma_{u}(b * \cup b)-\gamma_{u}(b *)
$$

where $b^{*}$ is the initial service $\mathrm{AP}$, and $\gamma_{u}\left(b_{*}\right)$ is the SINR of UE $u$ from AP $b^{*}$.

Given the maximum CAPG size $M$, the CAPG formation problem is formulated to maximize the $\mathrm{CB}$ for each UE:

$$
\begin{aligned}
& \max _{\mathcal{A}_{u}} \sum_{b \in \mathcal{A}_{u}}\left(v_{u, b}-\theta\right) \\
& \text { s.t. }\left|\mathcal{A}_{u}\right| \leq M \quad \forall u \in \mathscr{E}
\end{aligned}
$$

where $\mathcal{A}_{u}$ is the CAPG of UE $u, \theta$ is a given threshold of $\mathrm{CB}$.

The main idea of EU-CoMP method is to mitigate interference from the neighbor APs for edge UEs. To simplify the computational complexity, the APs with largest RSRP are selected as candidate coordinated set

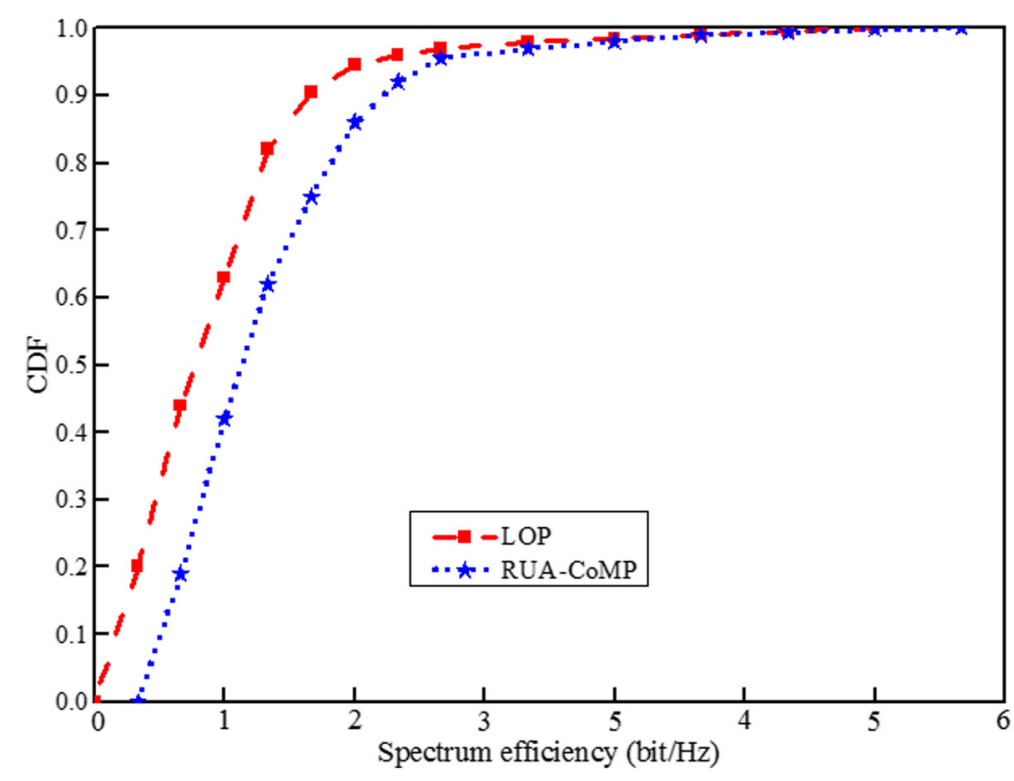

Fig. 3 Spectrum efficiency CDF 


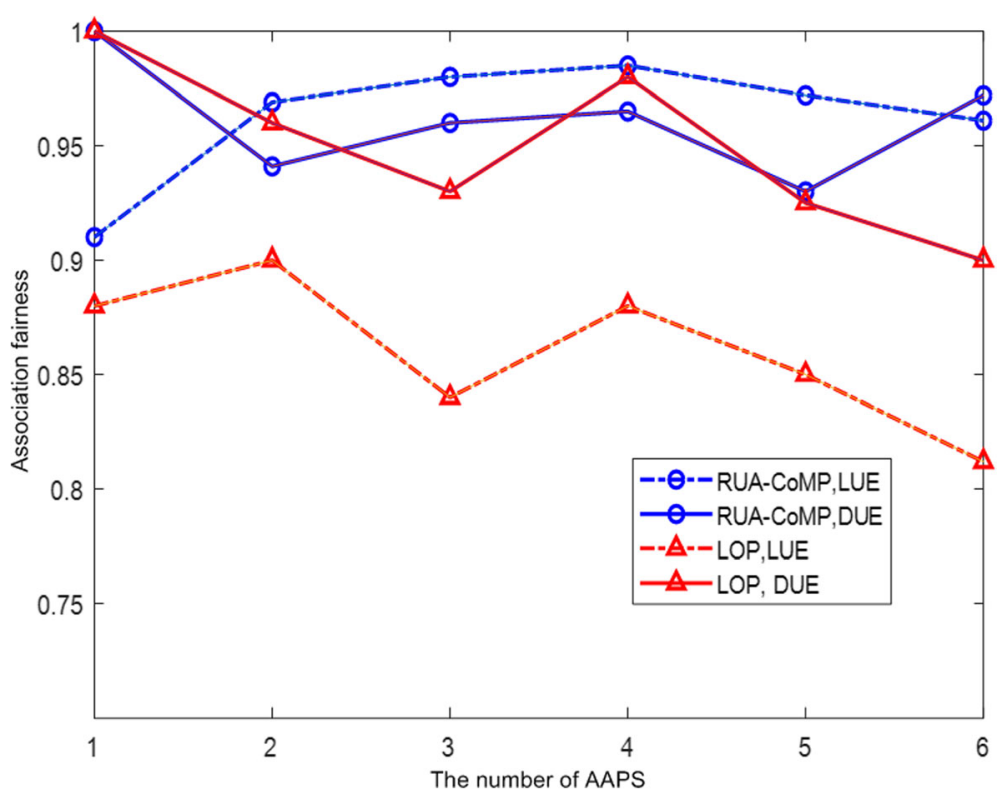

Fig. 4 User association fairness under different AAP numbers

(CCS), and the APs which are selected to form CAPG are all included in CCS.

A greedy heuristic algorithm is adopted to select the neighbor APs to form CAPG, and the steps are elaborated as follows:

Step 1: Select $M$ APs with the largest RSRP to form $\operatorname{CCS} \mathcal{C}_{u}$ for each edge UE $u$, initialize $\mathcal{A}_{u}=\Phi$.

Step 2: Find the $A P b$ with the largest SINR from $\mathcal{C}_{u}$, and calculate its CB $v_{u, n}$ according to Eq. (8).

If $v_{u, b}>\theta$, set $\mathcal{A}_{u}=\mathcal{A}_{u} \cup\{b\}$ and $C_{u}=C_{u} \backslash\{b\}$;

Otherwise break.
Step 3: If $\mathcal{C}_{u} \neq \Phi$, then go to step 2.

\section{Simulation results and discussion}

\subsection{Simulation settings}

The authors use MATLAB software as a simulation platform, in which a 3-tier drone-assisted heterogeneous scenario is considered. During the simulation, three LAPs are uniformly deployed within the coverage of the macro station, while the AAPs are flexibly deployed according to the UEs' QoS rate requirement. It is assumed that all AAPs are fixed at a height of $150 \mathrm{~m}$. The system

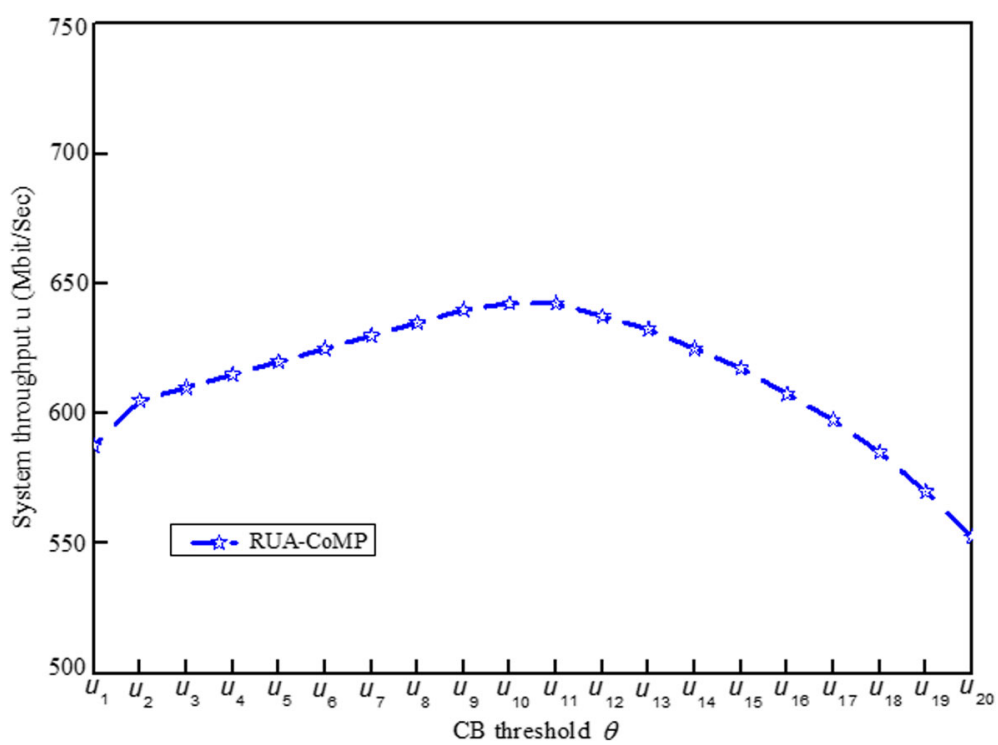

Fig. 5 System throughput under CB threshold variance 


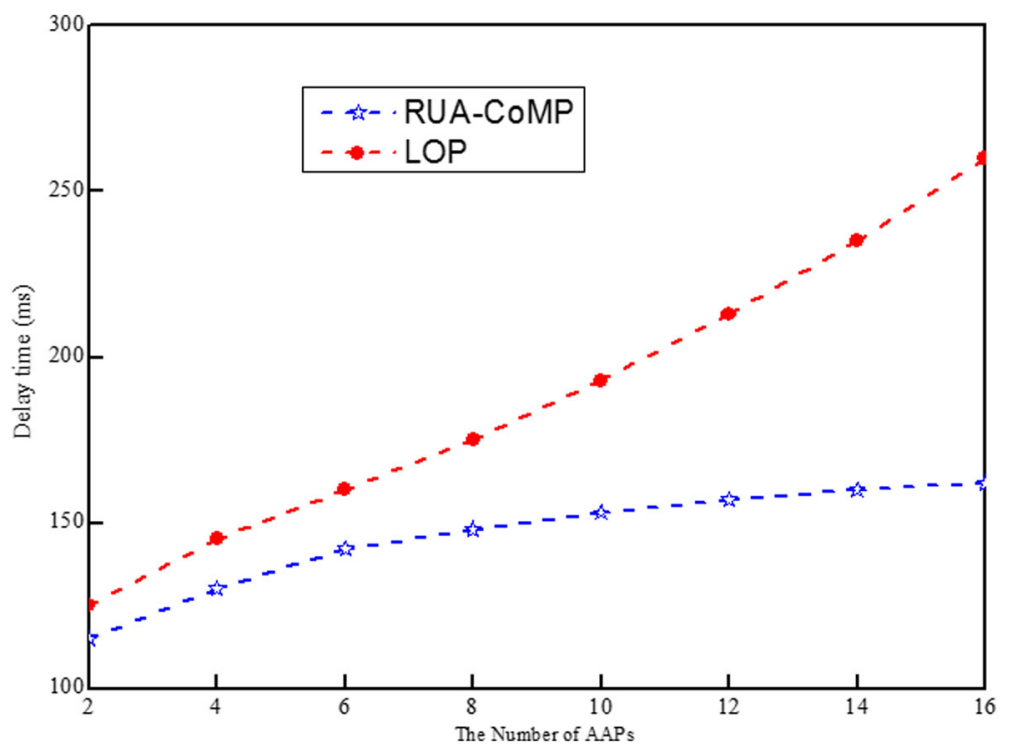

Fig. 6 Delay time under different AAP numbers

bandwidth is $10 \mathrm{MHz}$ and the frequency is $2.0 \mathrm{GHz}$. The main simulation parameters are summarized in Table 1.

\subsection{Numerical results and discussion}

In this simulation, the average data rate, JFI, and delay time are used to evaluate the performance of the proposed scheme. Note that the delay time is generally considered as the processing delay in the service APs determination. The performance of the proposed relevance user association with EU-CoMP guaranteeing (RUA-CoMP) scheme is compared with that of the largest load balancing scheme for user association problem (LOP) [10]. In LOP algorithm, the user association strategy is only based on the average received RSRP.

Figure 2 illustrates the average data rate performance of the two schemes at different AAP numbers. As seen in this figure, the average data rate increases as the number of AAPs increases, and the proposed scheme performs better than the LOP scheme. The reason is that the UEs are associated with the AP with the best RSRP in LOP scheme, which results in unequal UEs association between the three kinds of APs, diminishing the offloading capability of AAP and reducing the average data rate of UE. Moreover, with the increasing of minimum QoS rate requirement $r_{m}$, which means the higher transmission rate target of UEs, it will lead to an increase in the average data rate.

Figure 3 shows the UEs' Spectrum efficiency of the two schemes when the number of AAPs is 6. It can be seen that the performance curve of the proposed scheme is more on the right side than that of LOP algorithm, especially in the case of 5\% UEs with poor channel environment. The reason is that, compared with OPA scheme, the proposed scheme adopts EU-CoMP supporting approach to improve service performance of edge UEs.

Figure 4 illustrates the influence of different AAP numbers on the association fairness when $r_{m}=0.5 \mathrm{Mbps}$. It should be noted that the association fairness of the two schemes is compared under the same QoS rate requirement. As it is seen in this figure, with the increase of the number of AAPs, the JFI performance curves of both schemes fluctuate to a certain extent. This is due to the fact that UEs in the heavily loaded LAPs are unloaded into AAP, which improves the JFI of UEs in the LAP. When the number of AAP exceeds 4, only a small fraction of UEs are served by partial LAP. This may result in unequal user association between the two kinds of AAP and LAP. It should be noted that in this simulation, MAP only acts as the control entity and does not operate on UP.

Figure 5 plots the system throughput versus the threshold $\theta$ of CB for the case $M=4$. The value range of threshold $\theta$ is $\mathrm{E}=\left\{\mu_{1}, \mu_{2}, \cdots \mu_{u}\right\}$, where $\mu_{u}$ is the average CB of all APs in CCS $\mathcal{C}_{u}$, and we chose 20 UEs to test the threshold $\theta$ in this experiment. The values of $E$ are sorted in descending order. As can be seen from the figure system throughput increases first and then decreases as the threshold $\theta$ decreases. This is due to the fact that, when a little threshold is considered, more APs are selected to provide cooperative service for edge UE, which increases diversity gain. However, the cooperation among too many APs limits the reuse of certain resource for other UEs. At a certain CB threshold (around the average value of $E$ ), there is a balance between diversity and multiplexing. 
Figure 6 shows the delay time for user association of the two schemes. It should be noted that the delay time does not include the processing time of the UE accessing the AP. As can be seen in this figure, the delay time increases as the number of AAPs increases for two schemes. Compared with LOP scheme, the delay time in the proposed scheme increases relatively slowly and perform better.

\section{Conclusion}

In this letter, an intelligent user association scheme is proposed in the 3-tier DAHN. The proposed model uses association degree to decide the services AP for UEs, whereby the load of each AP and UE's SINR are jointly considered. For effectively tackling user association problem, we formulate it as a UE-AP association degree maximization problem. Due to the non-convexity of the optimization problem, variable relaxation is used to find the near-optimal solution. Further, to improve service performance of edge UEs, the EU-CoMP supporting approach is proposed. Simulation results show that the proposed scheme performs well in terms of service performance, user access fairness, and complexity.

\section{Abbreviations}

AAP: Aerial access point; CAPG: Coordinated access point group; CP: Control plane; DAHN: Drone-assisted heterogeneous networking; EU-CoMP: Edge user coordinated multiple points; LAP: Low-power access point; MAP: Macro access point; QoS: Quality of service; RSRP: Reference Signal Received Power; SINR: Signal-to-interference-plus-noise ratio; UE: User equipment; UP: User plane

\section{Acknowledgements}

The authors would like to thank the reviewers for their helpful advice.

\section{Authors' contributions}

Conceptualization and formal analysis, $\mathrm{CW}$ designed the experiment, $\mathrm{BH}$ methodology, SC. All authors read and approved the final manuscript.

\section{Funding}

This work was supported in part by the Key Project of University Outstanding Young Talents Project in Anhui under grant gxyqZD2018069.

\section{Availability of data and materials}

Mostly, I got the writing material from different journals as presented in the references. A MATLAB tool has been used to simulate my concept.

\section{Competing interests}

The authors declare that they have no competing interests.

\author{
Author details \\ ${ }^{1}$ State Key Lab of Networking and Switching Technology, Beijing University \\ of Posts and Telecommunications, Beijing 100081, China. ${ }^{2}$ School of \\ Information and Network Engineering, Anhui Science and Technology \\ University, Chuzhou 233100, China. ${ }^{3}$ State Key Lab of Wireless Mobile \\ Communication, China Academy of Telecommunication Technology, Beijing \\ 100191, China.
}

Received: 14 March 2019 Accepted: 29 May 2019

Published online: 17 June 2019

\section{References}

1. P. Kela, J. Turkka, M.Á. Costa, Borderless mobility in $5 \mathrm{G}$ outdoor ultra-dense networks. IEEE ACCESS 3, 1462-1476 (2015)
2. R. Han, C. Feng, H. Xia, in 2013 IEEE 24th International Symposium on Personal Indoor and Mobile Radio Communications (PIMRC). Optimal user association based on topological potential in heterogeneous networks (2013), pp. 2409-2413

3. N. Wang, E. Hossain, V.K. Bhargava, Joint downlink cell association and bandwidth allocation for wireless backhauling in two-tier HetNets with largescale antenna arrays. IEEE Trans Wirel Commun. 15(5), 3251-3268 (2016)

4. Y. Chen, J. Li, Z. Lin, G. Mao, B. Vucetic, User association with unequal user priorities in heterogeneous cellular networks. IEEE Trans Veh Technol. 65(9), 7374-7388 (2016)

5. T. Zhou, Y. Huang, W. Huang, S. Li, Y. Sun, L. Yang, in 2014 IEEE 80th Vehicular Technology Conference (VTC2014-Fall), Vancouver. QoS-aware user Association for Load Balancing in heterogeneous cellular networks (2014), pp. 1-5

6. J. Plachy, Z. Becvar, P. Mach, R. Marik, M. Vondra, Joint positioning of Flying Base stations and Association of Users: Evolutionary-based approach. IEEE Access 7, 11454-11463 (2019)

7. Y. Jin, L. Qiu, Joint user association and interference coordination in heterogeneous cellular networks. IEEE Commun Lett 17(12), 2296-2299 (2013)

8. Y. IKEDA, S. Okasaka, K. Higuchi, Joint optimization of user association and inter-cell interference coordination for proportional fair-based system throughput maximization in heterogeneous cellular networks. IEICE Trans Commun 10(8), 11-15 2017

9. Q. Kuang, W. Utschick, Energy management in heterogeneous networks with cell activation, user association, and interference coordination. IEEE Trans. Wirel. Commun. 15(6), 3868-3879 (2016)

10. J. Ben Abderrazak, A. Zemzem, H. Besbes, in 2015 6th International Conference on the Network of the Future (NOF), Montreal. QoS-driven user association for load balancing and interference management in HetNets (2015), pp. 1-3

11. A. Al-Hourani, S. Kandeepan, A. Jamalipour, in IEEE Global Communications Conference, Austin. Modeling air-to-ground path loss for low altitude platforms in urban environments (2014), pp. 2898-2904

12. S. Zhang, H. Zhang, B. Di, L. Song, Cellular UAV-to-X communications: Design and optimization for multi-UAV networks. IEEE Trans. Wirel. Commun. 18(2), 1346-1359 (2019)

13. J.G. Andrews, Seven ways that HetNets are a cellular paradigm shift. IEEE Commun. Mag. 51(3), 136-144 (2013)

14. M. Dianati, X. Shen, S. Naik, A new fairness index for radio resource allocation in wireless networks. Wirel Commun Netw Conf. 2, 712-717 (2005)

15. M.C. Ertürk, I. Güvenç, S. Mukherjee, H. Arslan, Fair and QoS-oriented resource management in heterogeneous networks. EURASIP J Wirel Commun Netw. 2013, 121 (2013)

16. Z. Huang, G. Shan, J. Cheng, J. Sun, TRec: An efficient recommendation system for hunting passengers with deep neural networks. Neural Comput Applic. (2018). https://doi.org/10.1007/s00521-018-3728-2

17. B. Wu, L. Zong, X. Yan, C. Guedes Soares, Incorporating evidential reasoning and TOPSIS into group decision-making under uncertainty for handling ship without command. Ocean Eng 39, 590-603 (2018)

\section{Publisher's Note}

Springer Nature remains neutral with regard to jurisdictional claims in published maps and institutional affiliations.

\section{Submit your manuscript to a SpringerOpen ${ }^{\circ}$ journal and benefit from:}

- Convenient online submission

- Rigorous peer review

- Open access: articles freely available online

High visibility within the field

- Retaining the copyright to your article

Submit your next manuscript at $>$ springeropen.com 